\title{
On-site number statistics of ultracold lattice bosons
}

\author{
Barbara Capogrosso-Sansone, ${ }^{1}$ Evgeny Kozik, ${ }^{1}$ Nikolay Prokof'ev, ${ }^{1,2}$ and Boris Svistunov ${ }^{1,2}$ \\ ${ }^{1}$ Department of Physics, University of Massachusetts, Amherst, MA 01003 \\ ${ }^{2}$ Russian Research Center "Kurchatov Institute", 123182 Moscow, Russia
}

\begin{abstract}
We study on-site occupation number fluctuations in a system of interacting bosons in an optical lattice. The ground-state distribution is obtained analytically in the limiting cases of strong and weak interaction, and by means of exact Monte Carlo simulations in the strongly correlated regime. As the interaction is increased, the distribution evolves from Poissonian in the non-interacting gas to a sharply peaked distribution in the Mott-insulator (MI) regime. In the special case of large occupation numbers, we demonstrate analytically and check numerically that there exists a wide interval of interaction strength, in which the on-site number fluctuations remain Gaussian and are gradually squeezed until they are of order unity near the superfluid (SF)-MI transition. Recently, the on-site number statistics were studied experimentally in a wide range of lattice potential depths [Phys. Rev. Lett. 96, 090401 (2006)]. In our simulations, we are able to directly reproduce experimental conditions using temperature as the only free parameter. Pronounced temperature dependence suggests that measurements of on-site atom number fluctuations can be employed as a reliable method of thermometry in both SF and MI regimes.
\end{abstract}

PACS numbers: 32.80.Pj, 39.90.+d, 67.40.Db

\section{INTRODUCTION}

Experiments on ultracold atoms trapped by an optical potential 1, 2, [3, 4, [5], offer a unique possibility to explore fundamental properties of strongly correlated quantum many-body systems allowing virtually unlimited control over the microscopic Hamiltonian parameters (see, e.g., [6] and references therein). System flexibility along with relatively long decoherence times puts it among top candidates for implementation of quantum information algorithms [] ]. In atomic interferometry [7], ultracold gases in the strongly correlated regime allow to achieve accuracies below the standard shot noise limit [8, 9]. A new exciting application, "atomtronics", is suggested by a remarkable analogy between the physics of ultracold atoms in optical lattices and that of electrons in crystals [10]. With the current experimental technique, it seems plausible to produce such basic atomtronic devises as diodes and bipolar junction transistors, which serve as building blocks for amplifiers and logic gates.

On the fundamental physics side, strongly interacting lattice bosons provide insight into the nature of quantum phase transitions. In particular, these systems are an accurate experimental realization of the Bose-Hubbard model [1],

$$
H=-t \sum_{<i, j>} a_{i}^{\dagger} a_{j}+\frac{1}{2} U \sum_{i} \hat{n}_{i}\left(\hat{n}_{i}-1\right)+\sum_{i} \epsilon_{i} \hat{n}_{i}
$$

where $\hat{n}_{i}=a_{i}^{\dagger} a_{i}$ is the number operator on site $i ; a_{i}^{\dagger}$ and $a_{i}$ respectively create and annihilate bosons on lattice sites, and $\langle i, j\rangle$ denotes the sum over nearest neighbors. The first term describes tunneling between neighboring potential wells of the optical lattice, the second term is the effective repulsion within a well, while the last term is due to an additional smooth space-varying potential, such as, e.g., a magnetic trap. This system exhibits a transition between superfluid (SF) and Mottinsulator (MI) groundstates governed by the competition of atom mobility and interatomic interaction [12. The SF-MI phase transition is a topic of intense current research both theoretically [13, 14, 15] and experimentally [16, 17, 18]. In typical experiments, a prepared BoseEinstein condensate of ultracold atoms is driven to the strongly correlated regime by means of its adiabatic loading into a periodic optical potential (optical lattice) induced by the a.c. Stark effect of interfering laser beams. The mobility of atoms, namely the hopping $t$, and their interaction $U$ are controlled by the depth of the optical potential, i.e. by the laser intensity. In relatively shallow potentials, atoms are delocalized over the entire lattice giving rise to long-range coherence and thus [19] to superfluid behavior.

If the lattice filling is commensurate, i.e. there is on average an integer number of atoms per lattice site, increasing the lattice depth brings the system across the phase transition to the MI state, which is characterized by zero compressibility and a gap in the spectrum of elementary excitations. Here, the key observable is the atom interference pattern obtained upon releasing the atoms and letting the atom cloud expand for a transient time of flight [1]. Phase correlations between lattice sites result in pronounced interference peaks smeared by the finite correlation length in the MI regime. At the same time phase coherence is fundamentally connected with the statistics of atom number fluctuations on lattice sites. In particular, one expects the on-site atom number to behave as a canonically conjugate variable with respect to the phase field and, therefore, experience suppressed fluctuations in the MI regime, analogous to the number squeezed states with sub-Poissonian number fluctuations [20] widely studied in quantum optics (see, e.g., 21]). In practice, number squeezed states are important for high-precision atomic interferometry [], where 
their use can potentially lead to sensitivities limited only by the Heisenberg uncertainty principle 8, 9, 22], and for atom-based quantum computing techniques [6], where unwanted number fluctuations necessitate correction procedures in operation of quantum gates.

Until recently, the number distribution was not measured directly. The situation changed with the development of the spin-oscillation technique [3], which is sensitive to the number of atom pairs and works at arbitrary lattice depths [17], and, most recently, the microwave spectroscopy using atomic clock shifts [4]. In Ref. [17], Gerbier et al. observed a drastic change of atom number statistics as the system of ${ }^{87} \mathrm{Rb}$ atoms was driven through the SF-MI transition. On the theoretical side, apart from the recent mean-field calculation [23], a comprehensive study of atom number fluctuations in the strongly correlated regime is still missing.

In the present work, we attempt to close this gap by tackling the problem both analytically and numerically. In section III we focus on the academic case of a homogeneous square lattice in the thermodynamic limit and in the limit of zero temperature in one-(1D), two-(2D) and three-(3D) dimensions. At $U=0$, the ground state of an ideal Bose gas is a Bose-Einstein condensate with characteristic Poisson distribution of number fluctuations. In subsection IA we consider the limit of weak interaction $\nu U / t \ll 1$, where $\nu$ is the filling factor. This parameter region corresponds to perturbative squeezing of the Poisson distribution, which, as a function of $\nu U / t$, qualitatively depends on the space dimension $d$. We consider the case of large filling factors $\nu \gg 1$ separately in subsection IIB because it is qualitatively different from that of $\nu \sim 1$. Indeed, the quantum nature of the SF-MI transition implies that the number fluctuations in the vicinity of the transition must be of order unity. At the same time, at $U=0$ the variance of the on-site number distribution is $\sigma^{2}=\nu \gg 1$. Therefore, there exists an extensive range of interactions (defined by the condition $U / t \lesssim \nu$ ), in which the system remains superfluid, but its on-site number distribution is drastically squeezed before the SF-MI can take place. We show that, at $U / t \ll \nu$, the on-site number statistics are Gaussian and derive the variance $\sigma^{2}$ of the distribution, which scales as $\sigma^{2} \propto \sqrt{\nu t / U}$ at $1 / \nu \ll U / t \ll \nu$ in all dimensions. In subsection $\amalg \mathrm{C}$ we suggest an expression that interpolates $P(n)$ between the limiting cases of small interaction and large occupation numbers, which is found to properly describe $P(n)$ up to $U / t$ of the order of the critical value $(U / t)_{c}$. The strong coupling limit, $\nu t / U \ll 1$, at integer filling is considered in subsection IID In this limit, the system is in the MI regime and the on-site number distribution is governed by rare particle-hole fluctuations.

We study the distribution in the strongly correlated regime connecting the limiting cases by means of a direct numeric simulation of the model (11) at $\nu=1$ in subsection IIE The distribution of the on-site occupation number is a local property, revealing no critical features at the transition. However, the strongly corre- lated region is responsible for the crossover that changes the statistics qualitatively. As the interaction strength is increased, we observe a gradual squeezing of the on-site number distribution and the emergence of the symmetry between particle- and hole-like fluctuations, characteristic of a MI. In this section, we also present numerical data for the case of large filling factors and demonstrate that the analysis of subsection IIB is applicable already at $\nu=5$.

The worm algorithm quantum Monte Carlo (MC) technique 24 allows us to simulate system sizes that are currently realized in experiments without any approximations, including the particle number. The results of a direct numeric simulation of the experimental setup of Ref. 17] are presented in section [II] With the lattice parameters fixed by the experiment, we are left with temperature as the only free parameter. Due to a smooth confining potential present on top of the optical lattice, the number distribution is not homogeneous. We focus on an integral characteristic of the number distribution, namely, the fraction of atoms found on lattice sites with occupation $n$, which can be systematically measured experimentally. This quantity has a pronounced temperature dependence in both SF and MI regimes.

The problem of thermometry in optical lattices, especially in the MI regime, is a long standing one. The ability to control the temperature is of crucial importance for applications that rely on the peculiar properties of a MI state. At $T=0$ fluctuations are of quantum nature and can be efficiently controlled externally through the lattice parameters, whereas temperatures comparable to the Mott excitation gap destroy the insulating state by activating particle-hole excitations. At the moment, there are no experimental techniques to measure the temperature of a strongly interacting system. Unlike in the weakly interacting regime, where the temperature can be straightforwardly extracted from the momentum distribution (e.g., from the interference pattern of matter waves or from the condensate fraction observed after the trap is released and the gas expands freely), in the strongly correlated regime, both temperature and interatomic interaction are responsible for filling the higher momentum states making standard absorption imaging techniques inapplicable.

The idea of using occupation number distributions to estimate the temperature was explored in Ref. [25], where in was argued that the temperature dependence of the total number of pairs and their spatial distribution (in traps) provides a sensitive method of thermometry deeply in the MI phase at energies smaller than the interatomic interaction, but much larger than the effective hopping between the sites. [Recently, it has become possible to directly sample spatially-resolved number distributions by spin changing collisions [16], microwave spectroscopy [4], and the scanning electron microscope [26] promising a complete practical realization of this method.] In this paper, we perform thermometry of the system in all strongly correlated regimes by comparing experimental 
data and numerical results for the statistics of occupation numbers. More specifically, we compare numerically calculated fraction of pairs $(n=2)$ with that measured in Ref. 17] across the SF-MI transition estimating the range of experimental temperatures. The accuracy of this method is mainly limited by the error bars of the experimental data and by the range of applicability of the Bose-Hubbard model.

\section{HOMOGENEOUS LATTICE}

In this section, we assume that there is no spacevarying potential on top of the optical lattice, and set $\epsilon_{i} \equiv \epsilon_{0}$. Let $N_{s}$ be the number of lattice sites and $N$ be the total number of particles. The goal of this section is to obtain the ground state probability $P_{n}$ to detect $n$ particles on a given lattice site in the limit of $N_{s}, N \rightarrow \infty$, at a fixed filling factor $\nu=N / N_{s}$. Mathematically, $P_{n}$ can be defined as

$$
P_{n}(U / t)=\sum_{\left\{n_{i \neq 1}\right\}}\left|\left\langle n_{1}=n,\left\{n_{i \neq 1}\right\}|| \Psi_{U / t}\right\rangle\right|^{2},
$$

where $\left|\Psi_{U / t}\right\rangle$ is the many-body ground state wavefunction, and $\left|\left\{n_{i}\right\}\right\rangle$ are Fock states.

\section{A. Weak coupling limit}

At sufficiently small $U / t$, the relevant representation of Eq. (1) is obtained by the diagonalization of the kinetic energy term with the canonical transformation $a_{i}=$ $N_{s}^{-1 / 2} \sum_{\mathbf{k}} b_{\mathbf{k}} \exp \left(i 2 \pi \mathbf{k} \mathbf{r}_{i} / L\right)$ (periodic boundary conditions are assumed), where $L=N_{s}^{1 / d}$ is the linear system size, $\mathbf{r}_{i}$ and $\mathbf{k}$ are respectively the position of the site $i$ and a quasi-momentum in $d$ dimensions with integervalued components, $-(L-1) / 2 \leq r_{i \mu}, k_{\mu} \leq(L-1) / 2$, $\mu=1, \ldots, d$. The result is

$$
H=\sum_{k} \varepsilon_{\mathbf{k}} b_{\mathbf{k}}^{\dagger} b_{\mathbf{k}}+\frac{U}{2 N_{s}} \sum_{\mathbf{k}_{1}+\mathbf{k}_{2}=\mathbf{k}_{3}+\mathbf{k}_{4}} b_{\mathbf{k}_{1}}^{\dagger} b_{\mathbf{k}_{2}}^{\dagger} b_{\mathbf{k}_{3}} b_{\mathbf{k}_{4}}
$$

with $\varepsilon_{\mathbf{k}}=2 t \sum_{\mu=1}^{d}\left[1-\cos \left(2 \pi k_{\mu} / L\right)\right]$. At $U=0$ the ground state of the Hamiltonian (3) is a pure BoseEinstein condensate, which can be expressed as a coherent state, $\left|\Psi_{(U / t)=0}\right\rangle=\exp \left(\sqrt{N} b_{0}^{\dagger}-N / 2\right)|0\rangle$. Then, transforming the wavefunction back to the on-site representation in terms of $\left\{a_{i}\right\}$ yields the Poisson distribution for the probability to find $n$ particles on a given site:

$$
P_{n}^{(0)}=e^{-\nu} \frac{\nu^{n}}{n !} .
$$

At a finite, but small, $U$ we employ the standard Bogoliubov method 27] of separating the system into the classical-field condensate part and non-condensate particles interacting with it, omitting the terms of the third and forth order with respect to the non-condensate operators. In this approximation, the Hamiltonian (3) is reduced to a bilinear in $b_{\mathbf{k}}$ and $b_{\mathbf{k}}^{\dagger}$ form and diagonalized by the canonical transformation $c_{\mathbf{k}}=u_{\mathbf{k}} b_{\mathbf{k}}+v_{\mathbf{k}} b_{-\mathbf{k}}^{\dagger}$, where

$$
\begin{gathered}
u_{\mathbf{k}}=\left[\left(\varepsilon_{\mathbf{k}}+\nu U\right) / 2 \omega_{\mathbf{k}}+1 / 2\right]^{1 / 2}, \\
v_{\mathbf{k}}=\left[\left(\varepsilon_{\mathbf{k}}+\nu U\right) / 2 \omega_{\mathbf{k}}-1 / 2\right]^{1 / 2}, \\
\omega_{\mathbf{k}}=\left[\varepsilon_{\mathbf{k}}^{2}+2 \nu \varepsilon_{\mathbf{k}} U\right]^{1 / 2} .
\end{gathered}
$$

The ground-state wavefunction is then obtained from the equation $c_{\mathbf{k}}\left|\Psi_{U / t}\right\rangle=0$ for all $\mathbf{k} \neq 0$ and has the form

$$
\left|\Psi_{U / t}\right\rangle=C \exp \left[\sqrt{N_{0}} b_{0}^{\dagger}-\frac{N_{0}}{2}-\frac{1}{2} \sum_{\mathbf{k} \neq 0} \frac{v_{\mathbf{k}}}{u_{\mathbf{k}}} b_{\mathbf{k}}^{\dagger} b_{-\mathbf{k}}^{\dagger}\right]|0\rangle,
$$

where $C$ is the normalization factor and $N_{0}=N-$ $\sum_{\mathbf{k} \neq 0}\left\langle b_{\mathbf{k}}^{\dagger} b_{\mathbf{k}}\right\rangle$ is the number of condensate particles.

Now we can express $\left|\Psi_{U / t}\right\rangle$ in terms of the on-site operators,

$$
\left|\Psi_{U / t}\right\rangle=C \exp \left[\sum_{i}\left(\sqrt{\nu_{0}} a_{i}^{\dagger}-\frac{\nu_{0}}{2}\right)-\sum_{i, j} S_{i j} a_{i}^{\dagger} a_{j}^{\dagger}\right]|0\rangle,
$$

with $\nu_{0}=N_{0} / N_{s}$ and

$$
S_{i j}=\frac{1}{2 N_{s}} \sum_{\mathbf{k} \neq 0} \frac{v_{\mathbf{k}}}{u_{\mathbf{k}}} e^{i 2 \pi \mathbf{k}\left(\mathbf{r}_{i}-\mathbf{r}_{j}\right) / L} .
$$

In this form, the wavefunction can be used to obtain the on-site number distribution in the whole range of $U / t \ll \nu$ by a straightforward application of Eq. (2).

We derive a closed-form expression for $P(n)$ in the limiting case of

$$
\alpha=\nu U / t \ll 1,
$$

which corresponds to the range of sufficiently weak squeezing of $P(n)$ allowing us to consider only the first correction to the Poisson distribution in the leading power of $\alpha$. Mathematically, Eq. (9) implies that $S_{i j} \ll$ 1. If, in addition, $S_{i j}$ is short-range, i.e. it decays at distances $\left|\mathbf{r}_{i}-\mathbf{r}_{j}\right| \sim 1$, which implies that the leading correction is insensitive not only to the system size, but also to the value of the healing length $\propto \alpha^{-1 / 2}$, then we can expand the exponential in Eq. (7) in powers of $S_{i j}$. Rather straightforward but lengthy algebra yields the distribution in the form

$$
P_{n}=P_{n}^{(0)}-\frac{\nu \lambda(\alpha)}{2}\left[P_{n}^{(0)}-2 P_{n-1}^{(0)}+P_{n-2}^{(0)}\right],
$$

where $\lambda$ is an interaction-dependent squeezing parameter and $P_{n}^{(0)}$ is given by Eq. (4) assuming $P_{n}^{(0)}=0$ for $n<0$. Identically,

$$
P_{n}=P_{n}^{(0)}\left(1+\frac{\lambda(\alpha)}{2}\left[\frac{n-(n-\nu)^{2}}{\nu}\right]\right) .
$$


Let us postpone writing an explicit expression for $\lambda$ and discuss the underlying assumptions leading to Eqs. (10), (11). It turns out that $S_{i j}$ is local only in 3D, where the main contribution to the integral in Eq. (8) comes from large momenta close to the edge of the Brillouin zone, $|\mathbf{k}| \sim L / 2$. In $2 \mathrm{D}$, the integral has a logarithmic divergency at low momenta in the limit $\alpha \rightarrow 0$, while, in $1 \mathrm{D}$, the dominant contribution to $S_{i j}$ comes from small momenta, meaning that in both $1 \mathrm{D}$ and $2 \mathrm{D}$ cases we can not rely on the perturbative expansion of the wave function. Nevertheless, Eqs. (10), (11) are still valid in 1D and $2 \mathrm{D}$, since the functional form of $P(n)$ must be the same in all dimensions. To prove this statement, we note that $P(n)$ is unambiguously determined by its characteristic function $\chi(t)=\left\langle\exp \left(i t a_{i}^{\dagger} a_{i}\right)\right\rangle$, which can be used to generate all moments of $P(n)$. The function $\chi(t)$ can be calculated explicitly as a series expansion in powers of $\left(i t a_{i}^{\dagger} a_{i}\right)$. Since averages of bosonic quasiparticle operators in the Bogoliubov theory obey Wick's theorem, each term in the series is a function of $\zeta \equiv\left\langle a_{i}^{\dagger} a_{i}\right\rangle$ and $\zeta^{\prime} \equiv\left\langle a_{i} a_{i}\right\rangle$. Therefore, $\chi(t)=\chi\left(t, \zeta, \zeta^{\prime}\right)$, meaning that all physical parameters, including the space dimension, enter $P(n)$ only through $\zeta$ and $\zeta^{\prime}$. In Eqs. (10), (11), this combination determines $\lambda(\alpha)$.

To determine $\lambda(\alpha)$ in all dimensions were note that it is directly related to the dispersion of $P(n)$ in Eq. (11)

$$
\sigma^{2} \equiv\left\langle(n-\nu)^{2}\right\rangle=\nu(1-\lambda) \text {. }
$$

On the other hand, $\sigma^{2}=\left\langle n_{i}^{2}\right\rangle-\nu^{2}=\left\langle a_{i}^{\dagger} a_{i} a_{i}^{\dagger} a_{i}\right\rangle-\nu^{2}$ can be calculated in a standard way by replacing $a_{i}$ with their expressions in terms of the Bogoliubov modes and the classical-field condensate part, i.e. $a_{i}=\sqrt{\nu_{0}}+$ $N_{s}^{-1 / 2} \sum_{\mathbf{k} \neq 0}\left[u_{k} c_{\mathbf{k}}-v_{k} c_{-\mathbf{k}}^{\dagger}\right] \exp \left(i 2 \pi \mathbf{k} \mathbf{r}_{i} / L\right)$. Then, an application of the Wick's theorem along with $\left\langle c_{\mathbf{k}} c_{\mathbf{k}^{\prime}}^{\dagger}\right\rangle=\delta_{\mathbf{k}, \mathbf{k}^{\prime}}$ and $\left\langle c_{\mathbf{k}} c_{\mathbf{k}^{\prime}}\right\rangle=0$ gives

$$
\sigma^{2}=\frac{\nu}{N_{s}} \sum_{\mathbf{k} \neq 0} \frac{\varepsilon_{\mathbf{k}}}{\omega_{\mathbf{k}}} .
$$

Strictly speaking, Eq. (13) is valid as long as the number of non-condensed particles is small (the 1D case is special in this regard and is further discussed below), $\left(N-N_{0}\right) / N \ll 1$, which implies $U / t \ll \nu^{(2-d) / d}$ at small $\nu$ and $U / t \ll \nu$ at large $\nu$. In the latter case, the distribution change can be non-perturbative since $\lambda \sim 1$ is typical in this parameter regime, which will be discussed in more detail in the next subsection.

Thus, we arrive at the following result

$$
\lambda=\frac{1}{N_{s}} \sum_{\mathbf{k} \neq 0}\left[1-\frac{\varepsilon_{\mathbf{k}}}{\omega_{\mathbf{k}}}\right] .
$$

The asymptotic behavior of $\lambda(\alpha \rightarrow 0)$ qualitatively depends on the dimensionality. In $1 \mathrm{D}$, the main contribution to the integral (14) comes from low momenta resulting in

$$
\lambda_{(d=1)}(\alpha) \rightarrow \frac{\sqrt{2}}{\pi} \sqrt{\alpha}
$$

In $3 \mathrm{D}$, there is no low-momentum singularity at $\alpha \rightarrow 0$, and the asymptotic expression is linear in $\alpha$ :

$$
\begin{gathered}
\lambda_{(d=3)}(\alpha) \rightarrow \frac{B}{2 \pi^{3}} \alpha, \\
B=\int_{0}^{\pi} \int_{0}^{\pi} \int_{0}^{\pi} \frac{d z_{1} d z_{2} d z_{3}}{\sum_{\mu=1}^{3}\left(1-\cos z_{\mu}\right)} \approx 15.673 .
\end{gathered}
$$

In $2 \mathrm{D}$, the low-momentum singularity is logarithmic

$$
\lambda_{(d=2)}(\alpha) \rightarrow \frac{\ln (C / \alpha)}{4 \pi} \alpha, \quad C \approx 23.54
$$

A comment is in order here concerning the derivation procedure for the $1 \mathrm{D}$ case. Formally, the condensate fraction is zero in the macroscopic limit even at $T=0$, while the derivation is based on the assumption that almost all the particles are Bose condensed. Nevertheless, our final results for the probabilities are valid even in $1 \mathrm{D}$, and the generic reasoning - based on the notion of quasicondensate - leading to this conclusion is as follows [28]. The quasicondensate correlation properties characteristic of a weekly interacting $1 \mathrm{D}$ gas at $T=0$ imply two different correlation radii, $r_{c}$ and $R_{c}, R_{c} \gg r_{c}$. Here $r_{c}$ defines the length scale upon which the system can be considered as macroscopic, while $R_{c}$ is the length at which (quantum) fluctuations of phase are of order unity. If the system size $L$ is such that

$$
r_{c} \ll L \ll R_{c}
$$

then the system is macroscopic with respect to all local properties, while still featuring a genuine condensate. (In a 1D weakly interacting system at $T=0$ the density of this condensate is close to the total density of particles.) Hence, for all local properties, including the ones discussed in the present paper, one can assume, without loss of generality, that the system size is finite and satisfies the condition (19). It should be emphasized that this assumption is implicit and is used exclusively to justify the derivation procedure. Otherwise, it does not lead to any explicit dependence of final answers on $L$. Indeed, the first inequality in Eq. (19) guarantees that all discrete sums over momenta can be replaced with integrals, and, since the integrals are convergent, the answer is independent of $L$.

\section{B. Large occupation number limit}

At $\nu \gg 1$ there exists a wide $(U / t \ll \nu)$ superfluid region, in which large number fluctuations given by Eq. (4) are gradually suppressed by the interaction until they become of order unity at the SF-MI transition. This physically appealing regime is not captured by Eqs. (10),(11), since they are applicable only for $U / t \ll 1 / \nu$.

At $\nu \gg 1$ and $U / \nu t \ll 1$, the number distribution is easily obtained due to the fact that the typical values 
of the occupation number fluctuations, $\eta_{i}=a_{i}^{\dagger} a_{i}-\nu$, are large $1 \ll\left|\eta_{i}\right| \ll \nu$. Thus, the transformation $a_{i}=\sqrt{\nu+\eta_{i}} \exp \left(i \Phi_{i}\right)$, where $\eta_{i}$ and $\Phi_{i}$ are canonically conjugate Hermitian operators, along with $\eta_{i} / \nu \ll 1$ reduces the Bose-Hubbard Hamiltonian (1) to

$$
\begin{array}{r}
H=-t \nu \sum_{<i, j>}\left[1+\frac{1}{4 \nu^{2}}\left(\eta_{i} \eta_{j}-\eta_{i}^{2}\right)\right] \\
\cos \left(\Phi_{i}-\Phi_{j}\right) \\
+\frac{U}{2} \sum_{i} \eta_{i}^{2}
\end{array}
$$

where $0 \leq \Phi_{i} \leq 2 \pi$. In fact, in this form, Eq. (20) is applicable at any $\eta_{i} \ll \nu$ including the description of the SF-MI transition and the MI phase. At sufficiently large interaction, namely $U / t \gg 1 / \nu$, the $\propto \eta_{i}^{2} / \nu^{2}$ term in Eq. (20) can be neglected, and the Hamiltonian coincides with the well-studied quantum rotor model (QRM).

In this work, we are interested in the $U / t \ll \nu$ limit of model Eq. (20), i.e. when phase fluctuations between the nearest-neighbor sites are small $\left|\Phi_{i}-\Phi_{j}\right| \ll 1$ and the number fluctuations are large. This allows us to consider $\eta_{i}$ as a continuous variable and formally redefine the domain of $\eta_{i}, \Phi_{i}$ as $-\infty<\eta_{i}, \Phi_{i}<\infty$. The result is a harmonic approximation of the Hamiltonian (20),

$$
\begin{aligned}
H=t \nu \sum_{<i, j>}\left[\frac{1}{2}\left(\Phi_{i}-\Phi_{j}\right)^{2}+\right. & \left.\frac{1}{4 \nu^{2}}\left(\eta_{i}^{2}-\eta_{i} \eta_{j}\right)\right] \\
& +\frac{U}{2} \sum_{i} \eta_{i}^{2}
\end{aligned}
$$

From its functional (quadratic) form we immediately conclude that the distribution of $\eta_{i}$, which is Gaussian at $U=0(\nu \rightarrow \infty$ limit of Eq. (4) $)$, remains Gaussian in a wide range of coupling strength - all the way to the vicinity of the SF-MI transition where $\sigma^{2} \sim 1$ and the model (21) breaks down. The proof is as follows. Since the Hamiltonian (21) is bilinear in $\left\{\eta_{i}, \Phi_{i}\right\}$, all averages are subject to the Wick's theorem. Therefore, the characteristic function of the distribution $W\left(\eta_{i}\right)$, which is given by the integral $\int_{-\infty}^{\infty} \exp \left(i k \eta_{i}\right) W\left(\eta_{i}\right) d \eta_{i}$, is a Gaussian $\exp \left(-k^{2} \sigma^{2} / 2\right)$.

The only parameter of the distribution, $\sigma^{2}$, is given by

$$
\sigma^{2}=\frac{\nu}{N_{s}} \sum_{\mathbf{k}} \frac{\varepsilon_{\mathbf{k}}}{\omega_{\mathbf{k}}}\left[1+2\left\langle c_{\mathbf{k}}^{\dagger} c_{\mathbf{k}}\right\rangle\right]
$$

where $c_{\mathbf{k}}$ and $c_{\mathbf{k}}^{\dagger}$ are the creation and annihilation operators of the normal modes of the Hamiltonian (21) with frequencies $\omega_{\mathbf{k}}$ given by Eq. (5). The Hamiltonian is di- agonalized by the transformation

$$
\begin{gathered}
\eta_{i}=\sum_{\mathbf{k}} \sqrt{\frac{\omega_{\mathbf{k}}}{2 U+\varepsilon_{\mathbf{k}} / \nu}}\left[\chi_{\mathbf{k} i} c_{\mathbf{k}}+\chi_{\mathbf{k} i}^{*} c_{\mathbf{k}}^{\dagger}\right], \\
\Phi_{i}=-i \sum_{\mathbf{k}} \sqrt{\frac{\omega_{\mathbf{k}}}{2 \nu \varepsilon_{\mathbf{k}}}}\left[\chi_{\mathbf{k} i} c_{\mathbf{k}}-\chi_{\mathbf{k} i}^{*} c_{\mathbf{k}}^{\dagger}\right], \\
\chi_{\mathbf{k} i}=\frac{1}{\sqrt{N_{s}}} \exp \left(i 2 \pi \mathbf{k} \mathbf{r}_{i} / L\right)
\end{gathered}
$$

Here, we restrict ourselves to ground state properties only and thus set $\left\langle c_{\mathbf{k}}^{\dagger} c_{\mathbf{k}}\right\rangle \equiv 0$, which leads exactly to Eq. (13). This is hardly surprising, since the model (21) is equivalent to the Bogoliubov approximation of the Hamiltonian (3) in the limit of large $\nu$, and thus, we could formally demonstrate that $P(n)$ is Gaussian at $\nu \gg 1$ already in the framework of section $\amalg A$ However, the hydrodynamic approach chosen in this section seems more natural and physically transparent when dealing with a dense system.

Let us examine properties of the distribution variance in more detail. An explicit expression for $\sigma^{2}$ reads

$$
\sigma^{2}=\frac{\nu}{\pi^{d}} \int_{0}^{\pi} \cdots \int_{0}^{\pi} \sqrt{\frac{\sum_{\mu}\left(1-\cos x_{\mu}\right)}{\nu U / t+\sum_{\mu}\left(1-\cos x_{\mu}\right)}} d x_{1} \cdots d x_{d},
$$

In the limit of $\alpha=\nu U / t \rightarrow 0$, this expression reduces to Eqs. (15)-(18). In the opposite limit, we find

$$
\sigma^{2}=\frac{I_{d}}{\pi^{d}} \sqrt{\frac{\nu t}{U}}, \quad U / t \gg 1 / \nu,
$$

where $I_{1}=2 \sqrt{2}, I_{2} \approx 13.373$, and $I_{3} \approx 52.348$. The latter formula allows to estimate the range of $U / t$ at which Eq. (24) is applicable. The condition $\sigma^{2} \gg 1$ gives $U / t \ll \nu$, consistent with the applicability range of Eq. (13).

\section{Interpolation formula in the SF regime}

In sections $\llbracket \mathrm{A}$ and $\amalg \mathrm{B}$ we have derived asymptotically exact solutions describing week squeezing $(\lambda \ll 1)$ and strong squeezing $(0 \leq \lambda \lesssim 1)$ at $\nu \gg 1$ respectively. The two limits overlap, which suggests that one can write a single interpolation formula to capture both limits correctly. This formula is also expected to predict $P(n)$ correctly in a broader region of $U / t$ even at $\nu \sim 1$. Formally, we have to find a function $\tilde{P}(n, \nu, \lambda)$ such that (i) it coincides with Eq. (11) in the limit of $\lambda \ll 1$, (ii) it becomes Gaussian as a function of $n$ at $\nu \gg 1$ and $0 \leq \lambda<1$ with the average $\nu$ and variance $\tilde{\sigma}^{2}=\nu(1-\lambda)$, and (iii) it is analytic with respect to $\nu$ and $\lambda$. The solution is not unique, and we simply suggest one which satisfies the above mentioned criteria

$$
\begin{aligned}
& \tilde{P}(n, \nu, \lambda)=c P^{(0)}(n) \\
& \quad \exp \left[\frac{\lambda(n-\nu)+\left(n-\nu^{2}\right)}{2 \nu}-\frac{(n-\nu)^{2}}{2 \nu(1-\lambda)}\right],
\end{aligned}
$$


where $c$ is the normalization factor and $P^{(0)}(n)$ is the Poisson distribution. By comparing Eq. (26) with numerical simulations at $\nu=1$ (described below) we find that this formula accurately describes the actual form of $P(n)$ in a much broader range of $U / t$ than the solution (11).

\section{Strong coupling limit}

Let us now turn to the strong coupling limit, $\nu t / U \ll$ 1 , at integer $\nu$, when atoms are well in the Mott insulator regime, and the zeroth order approximation to the wavefunction is a direct product of local Fock states, $\left|\Psi_{(t / U)=0}\right\rangle=\left|\left\{n_{i}=\nu\right\}\right\rangle$. The effect of finite hopping $t$ can be taken into account as a perturbation of the on-site interaction term in the Hamiltonian (1). To the first approximation, this results in an admixture of the particlehole pairs to $\left|\Psi_{(t / U)=0}\right\rangle$,

$$
\left|\Psi_{t / U}\right\rangle \propto\left(1+\frac{t}{U} \sum_{<i, j>} a_{i}^{\dagger} a_{j}\right)\left|\Psi_{(t / U)=0}\right\rangle, \quad \nu t / U \ll 1 .
$$

With this wavefunction Eq. (2) leads to the following distribution

$$
\begin{gathered}
P_{\nu}=1-2 P_{\nu-1}, \\
P_{\nu-1}=P_{\nu+1}=2 d \nu(\nu+1) t^{2} / U^{2}, \\
P_{n}=0, \text { if }|n-\nu|>1 .
\end{gathered}
$$

\section{E. Numerics}

Clearly, as $U$ is changed from 0 to $\infty$ the number distribution must change qualitatively. Having an essentially long tail at $n>\nu$ in the weakly interacting limit, $P_{n}$ becomes sharply peaked at large $U$ with equal probabilities for $\nu-1$ and $\nu+1$ particles on a site. To obtain $P_{n}(U / t)$ in the crossover regime, $t \sim U$, we perform $\mathrm{MC}$ simulations using the continuous-time Worm Algorithm scheme 24]. We set $\nu=1$ and take the limit $N_{s} \rightarrow \infty$, $\beta=1 / T \rightarrow \infty$, where $T$ is the temperature. For the linear system size $L=N_{s}^{1 / d}=24$ and $\beta \gg L / 2 \pi c$, where $c \sim 6 t$ is the typical value of sound velocity in the superfluid phase (higher temperatures can be used in the MI phase with gaps $\sim U$ ), the shape of the distribution is already well saturated, within a fraction of one percent in $3 \mathrm{D}$ and $2 \mathrm{D}$, and within a few percent in $1 \mathrm{D}$, consistent with the fact that $P_{n}$ is an essentially local characteristic.

The simulation results for $3 \mathrm{D}, 2 \mathrm{D}$ and $1 \mathrm{D}$ are shown in Fig. [1 where $P_{0}, P_{1}$ and $P_{2}$ are plotted as functions of $U / t$ along with the predictions of Eq. (26) and Eq. (28). The main observation is that, although $P_{n}(U / t)$ fundamentally does not reveal any critical behavior, in 3D and $2 \mathrm{D}$ the SF-MI transition is marked by a substantial change in $P_{n}$ curves - they rapidly plateau in the Mott regime for $U / t \gtrsim(U / t)_{c}$. As expected, in $1 \mathrm{D}$ the curves are much more smooth and the saturation at high $U$ is not pronounced. Remarkably, Eq. (26), deviates notably (by a few percent) from the numerical data only at $U / t$ as high as $\sim 2$ in $1 \mathrm{D}, \sim 4$ in $2 \mathrm{D}$, and $\sim 8$ in $3 \mathrm{D}$.

We compare the theoretical predictions of section for the asymptotics in the $\nu \gg 1$ limit with the results of Monte-Carlo simulations. From Fig. 2 where the simulated probability distribution at $U / t=2$ is plotted along with Gaussian curves with variances calculated by Eq. (24) at corresponding $\nu$, we conclude that the shape of the distribution is perfectly well (within the error bars) described by the Gaussian distribution already at $\nu=5$. In Fig. 31 the numerically simulated $\sigma^{2}$ and the prediction of Eq. (24) are plotted together as a function of $U / t$.

\section{COMPARISON WITH EXPERIMENT: EFFECTS OF FINITE TEMPERATURE}

In actual experiments, atoms are confined in the lattice by a trapping potential, typically of a parabolic form. This results in an inhomogeneous density profile in the SF regime and in the formation of Mott-plateaus [4, 16, 17] spherical shells of integer filling - in deep lattices. Correspondingly, the distribution of the number fluctuations is also inhomogeneous, i.e. $P_{n}=P_{n}(i)$, where $i$ is the site index. The development of experimental techniques allowing detection of atoms with a single-site spatial resolution [26] should open an exciting possibility to directly measure atom correlation functions and $P_{n}(i)$ in particular. Current experiments [4, 16, 17] typically deal with integral characteristics of the number distribution, such as the fraction of the total number of atoms found on lattice sites with occupation $n$,

$$
f_{n}=\sum_{i} n P_{n}(i)
$$

The fraction of pairs $f_{2}$ in both SF and MI regimes can be accurately probed by the spin-changing collisions 3 . The measurement is set up in the following way [17]. After the system is allowed to equilibrate at a fixed value of the lattice potential depth $V_{0}$, the configuration of atoms is frozen by a rapid increase of $V_{0}$. Then, coherent spin dynamics in the two-particle channel can be induced with a near-unitary efficiency, and the spin oscillation amplitude is measured to yield the fraction of pairs. With this technique, Gerbier et al. 17] observed $f_{2}$ as the system was driven from a SF regime across the transition deep into MI regime, corresponding to the values of the initial lattice potential $V_{0}$ ranging from $4 E_{r}$ to $40 E_{r}$, where $E_{r}=h^{2} / 2 m \lambda^{2}$ is the single photon recoil energy, $m$ is the mass of a ${ }^{87} \mathrm{Rb}$ atom, and $\lambda$ is the lattice laser wavelength.

In our simulations, the system of ${ }^{87} \mathrm{Rb}$ atoms of Ref. 17] is described by the Bose-Hubbard model (11). The external potential is harmonic, $\epsilon_{i}=m \omega_{0}^{2} \mathbf{r}_{i}^{2} / 2$, and all the parameters of the Hamiltonian (11) are fixed by 

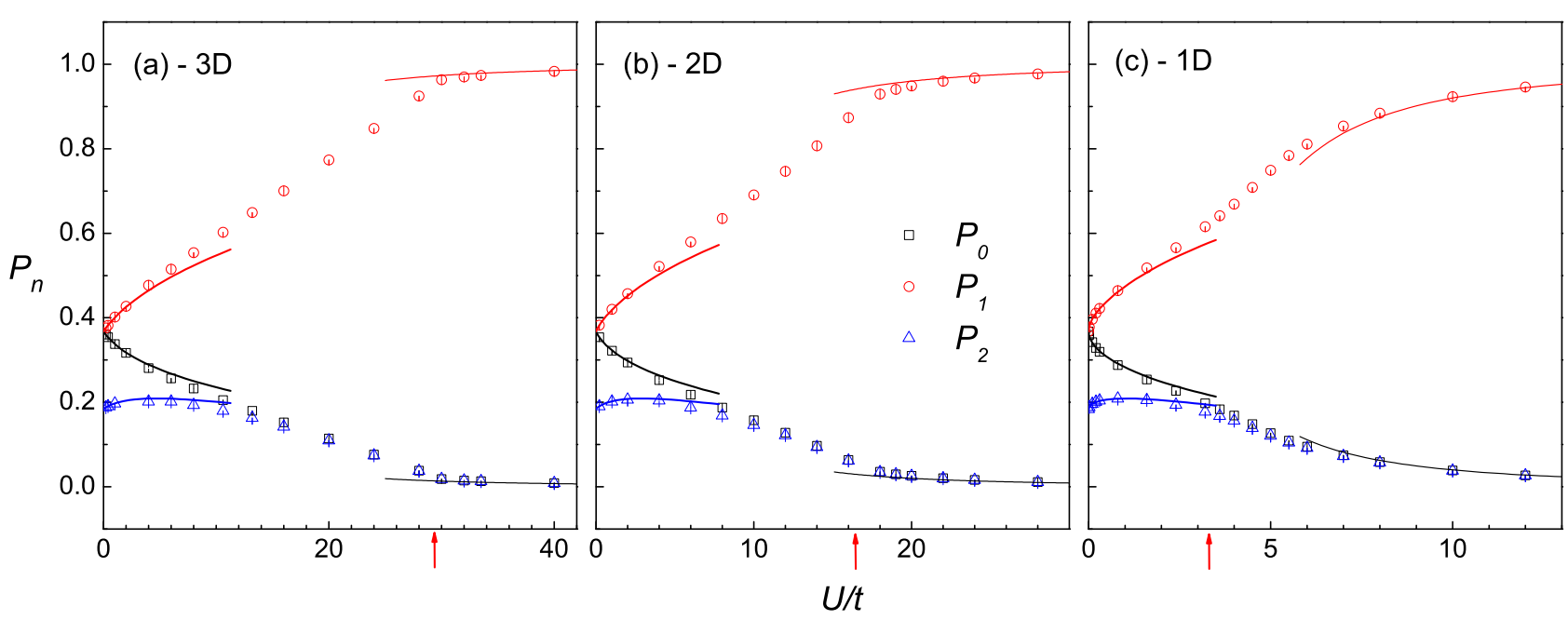

FIG. 1: (Color online.) The probability $P_{n}$ to detect $n$ particles on a single site of a homogenous square lattice as a function of $U / t$ at zero temperature and unitary filling. The results of MC simulation are shown for $n=0$ (squares), $n=1$ (circles) and $n=2$ (triangles). The uncertainties in $P_{n}$ are smaller than the symbol size. The predictions of Eq. (26) in the SF regime and that of Eq. (28) in the strong coupling limit are plotted by thick and thin solid lines respectively. The critical points of the SF-MI transition, marked by the arrows on the graph, are $(U / t)_{c}=29.34(2)$ in three dimensions $[29](\mathrm{a}),(U / t)_{c}=16.4(8)$ in two dimensions [30] (b), and $(U / t)_{c}=3.30(2)$ in one dimension [31] (c).

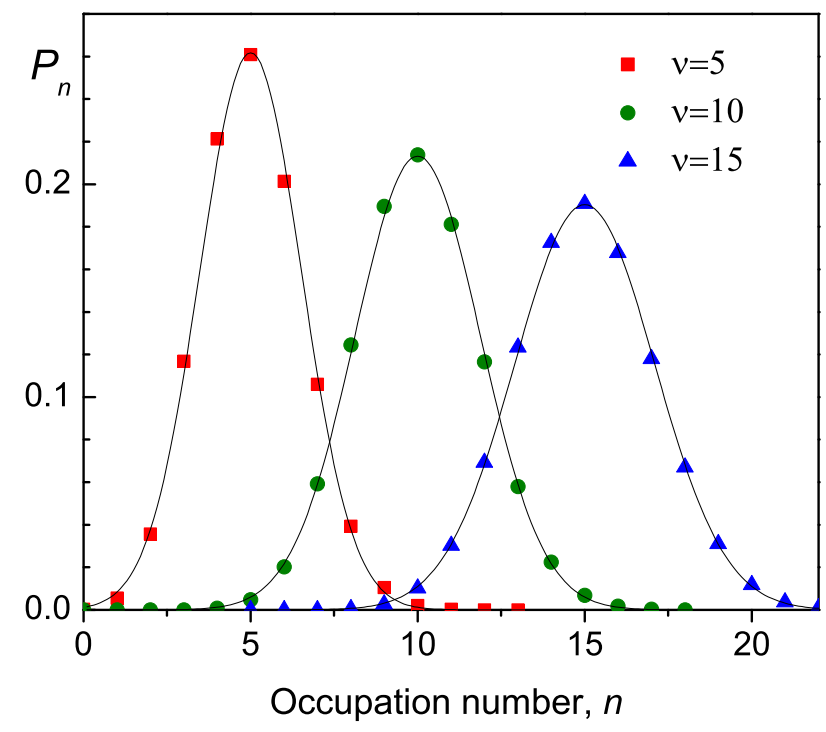

FIG. 2: (Color online.) Probability $P_{n}$ of the on-site occupation number $n=\eta_{i}+\nu$ at $U / t=2$ from the Monte-Carlo simulation (the error bars are smaller then the symbol size) with the filling factor 5 (squares), 10 (circles), and 15 (triangles). The solid lines are the Gaussian curves with the variances calculated from Eq. (24) with the parameters of simulations.

the experimental setup. We study the number fluctuations at two values of the lattice depth, $V_{0}=8 E_{r}$ and $V_{0}=13 E_{r}$ (corresponding to $U / t \approx 7.4$ and $U / t \approx 35.6$ respectively), which serve as examples of typical $\mathrm{SF}$ and MI phases in the strongly correlated regime. The trapping frequency $\omega_{0}$ is equal to $2 \pi \times 37 \mathrm{~Hz}$ and $2 \pi \times 46 \mathrm{~Hz}$ for $V_{0}=8 E_{r}$ and $V_{0}=13 E_{r}$ correspondingly 17,33 . We

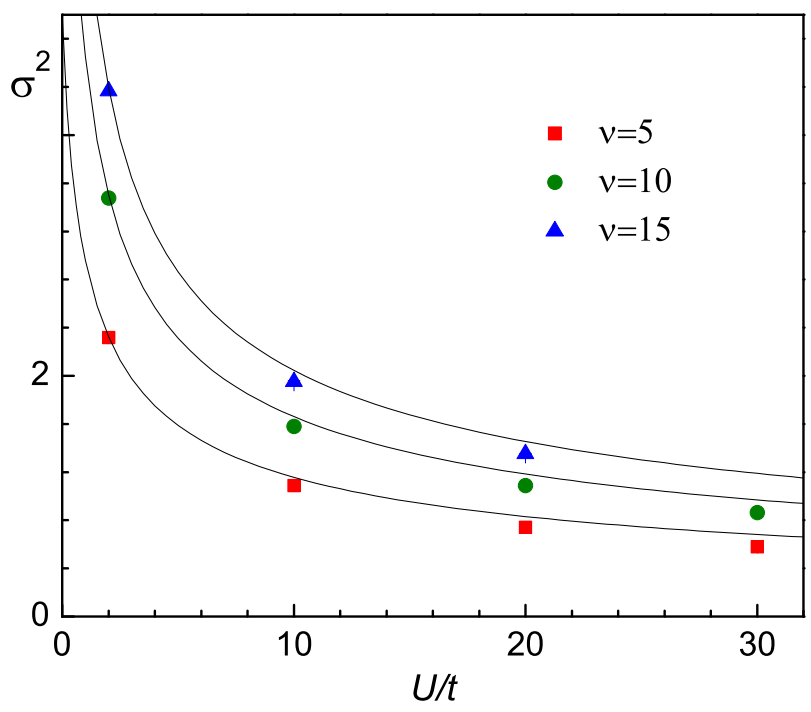

FIG. 3: (Color online.) The variance $\sigma^{2}$ as a function of interaction strength $U / t$ from the Monte-Carlo simulation (the error bars are smaller then the symbol size) with the filling factor 5 (squares), 10 (circles), and 15 (triangles). The solid lines represent the prediction of Eq. (24) with the parameters of simulations.

perform simulations in a sensible range of temperatures. Direct comparison between experimental and numerical data enables us to estimate the final temperature of the system. The results of the simulations are sown in Fig. [4 where the curves for $f_{1}, f_{2}$, and $f_{3}$ as functions of the total atom number in the trap $N$ are parameterized by the temperature.

Thermal effects vanish and a system can be considered 


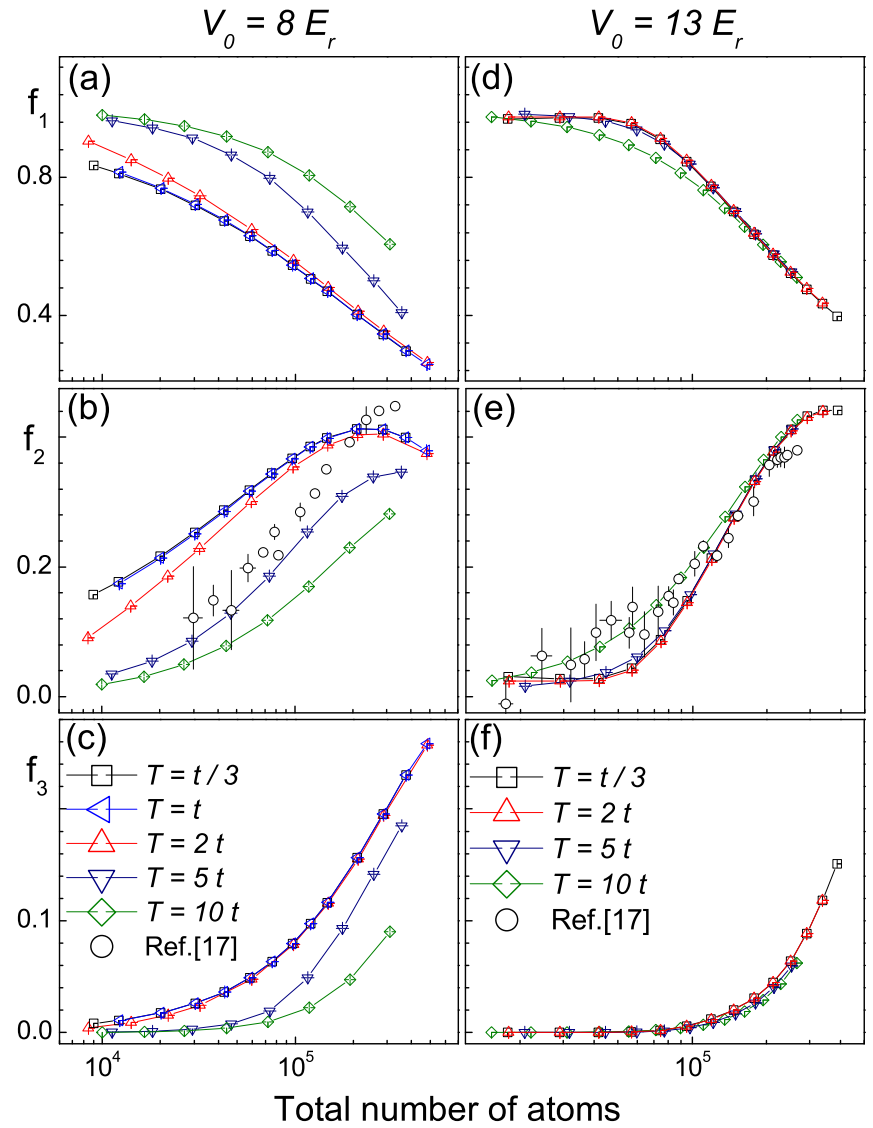

FIG. 4: (Color online.) Fractions of atoms $f_{1}, f_{2}, f_{3}$ occupying lattice sites with one, two and three particles on them respectively versus the total number of particles in the trap at the lattice depths $V_{0}=8 E_{r},(\mathrm{a}),(\mathrm{b}),(\mathrm{c})$, and $V_{0}=13 E_{r}$, (d), (e), (f). The numerical results at different temperatures are plotted with the experimental data for $f_{2}$ of Ref. [17].

to be in its ground state, if the temperature is substantially smaller than the energy of the low-lying excitations. In the SF regime, these modes have typical frequencies of order $\sqrt{m / m_{*}} \omega_{0}\left(m^{*}\right.$ is the effective mass in the lattice), which for the parameters of Fig. 目(a)-(c), gives a rough estimate $T \ll 3 t$. Clearly, the curves for $T=t / 3$ and $T=t$ in Fig. 世(a)-(c) are in the regime of effective zero temperature. Already at $T=2 t$ the thermal effects become significant resulting in a larger atom cloud and, consequently, reduced average density. At $T<5 t$ the size of the cloud for the maximum number of atoms in the trap is $\sim 100$ lattice sites in each dimension. To obtain $f_{n}$ at $T=10 t$, we have to resort to finite-size scaling, being limited by computer memory at the linear system size of 150 .

In the MI phase, the zero temperature regime is reached for temperatures smaller than the excitation gap, which is of order $U / 2$ for the parameters of Fig. 过(d)-(f). This leads to the condition $T \ll 20 t$, consistent with the saturation of $f_{n}$ below $T=5 t$ (see Fig. 廿1 d)-(f)). For $N \lesssim 5 \times 10^{4}$, in the zero temperature limit, the curves $f_{n}$ are flat, corresponding to the filling of the $\nu=1$ Mott shell, and the number distribution is essentially squeezed. Simple estimates [17] show that the decrease of $f_{1}$ accompanied by the increase in $f_{2}$ at $N \sim 5 \times 10^{4}$, and the saturation of $f_{2}$ with a peak in $f_{3}$ at $N \sim 2 \times 10^{5}$ are consistent with the formation of Mott plateaus with $\nu=2$ and $\nu=3$, respectively. As seen from Fig. 4 (d),(e), final temperature effects degrade the degree of number squeezing in the MI by favoring particle-hole excitations.

The comparison of the calculated fraction of atom pairs $f_{2}$ with the measurements of Ref. [17] (open circles in Fig. 廿(b),(e)) gives the typical experimental temperatures of order of $5 t \approx 1.5 \times 10^{-1} E_{r}$ in the $\mathrm{SF}$ regime and $10 t \approx 10^{-1} E_{r}$ in the MI regime. Temperatures on the order of a few $t$ have been also observed in a (onedimensional) Tonks-Girardeau gas 32, where the effective fermionization due to strong interactions allows to deduce the experimental temperature from the momentum distribution. Note that the system acquires a finite temperature as a result of its loading into the optical lattice, and therefore the final temperature is supposed to depend on the number of atoms in the trap. However, the fact that the experimental data lie above the $T=0$ curve in Fig. 4(b) at high $N$ is unlikely to be explained by the the effects of heating. When a large $f_{3}$ fraction is present, a change in the spin resonance condition can result in a considerable contribution of spin collisions on triply occupied sites to the observed spin oscillation amplitude [17], which could explain the anomalously high apparent $f_{2}$ 33]. Such drifts of the resonance parameters are carefully checked for, but can not be ruled out completely [33].

\section{CONCLUSIONS}

We studied the ground-state on-site number statistics of interacting lattice bosons. We considered the limits of weak interatomic interactions, the limit of large filling in the SF regime, and the limit of strong interatomic interactions. At $\nu U / t \ll 1$, the correction to the Poisson distribution is described by Eq. (11), with an essentially dimension-dependent scaling-Eq. (15) in 1D, Eq. (18) in 2D, and Eq. (16) in 3D. In the case of large occupation numbers, $\nu \gg 1$, we show that, in the region of interactions $U / t \ll \nu$, the on-site occupation number distribution is Gaussian and its variance, given by Eq. (24), gradually decreases with the asymptotic form $\sigma^{2} \propto \sqrt{\nu t / U}$ at $1 / \nu \ll U / t \ll \nu$ in all dimensions. An excellent agreement with numeric simulations is found already at $\nu=5$. At $\nu t / U \ll 1$ and integer filling $\nu$, the distribution, Eq. (28), is dominated by particle-hole fluctuations on top of an ideal MI. At $\nu=1$, we performed Monte Carlo simulations of the on-site occupation number distribution in $1 \mathrm{D}, 2 \mathrm{D}$, and $3 \mathrm{D}$ in a wide range of $U / t$ covering the crossover region between the limiting cases.

We simulated a parabolically confined system in the realistic case of Ref. 17] with the final temperature of the system being the only free parameter. By direct 
comparison between experimental data and numerical results at different temperatures, we were able to estimate the experimental temperature, which we found to be of the order of a few $t$ near the transition. The error bars are small enough to determine $T$ with accuracy of order $t$. Our results suggest that measurements of the on-site atom number fluctuations can serve as a reliable method of thermometry in both superfluid and Mott-insulator regimes. We believe that with more elaborate techniques, such as that giving access to the spatial number distribution $P_{n}(i)$, the temperature resolution can be further improved.

We are grateful to Fabrice Gerbier for providing us with the experimental parameters and for fruitful discussions of the results of this work. This research was supported by the National Science Foundation under Grant No. PHY-0426881.
[1] M. Greiner, O. Mandel, T. Esslinger, T.W. Hänsch, and I. Bloch, Nature 415, 39 (2002).

[2] S. Ospelkaus, C. Ospelkaus, O. Wille, M. Succo, P. Ernst, K. Sengstock, and K. Bongs, Phys. Rev. Lett. 96, 180403 (2006).

[3] A. Widera, F. Gerbier, S. Foelling, T. Gericke, O. Mandel, and I. Bloch, Phys. Rev. Lett. 95, 190405 (2005).

[4] G. K. Campbell, J. Mun, M. Boyd, P. Medley, A. E. Leanhardt, L. Marcassa, D. E. Pritchard, and W. Ketterle, cond-mat/0606642 (2006).

[5] T. Gericke, F. Gerbier, A. Widera, S. Foelling, O. Mandel, and I. Bloch, cond-mat/0603590 (2006).

[6] D. Jaksch and P. Zoller, Ann. Phys. 315, 52 (2005).

[7] P. Berman Ed., Atom Interferometry (Academic Press, New York, 1997).

[8] J. A. Dunningham, K. Burnett and S. M. Barnett, Phys. Rev. Lett 89, 150401 (2002); J. A. Dunningham and K. Burnett, Phys. Rev. A, 70, 033601 (2004).

[9] M. Rodríguez, S. R. Clark, and D. Jaksch, condmat/0607397 (2006).

[10] B. T. Seaman, M. Krämer, D. Z. Anderson, and M. J. Holland, cond-mat/0606625 (2006).

[11] D. Jaksch, C. Bruder, J.I. Cirac, C.W. Gardiner, and P. Zoller, Phys. Rev. Lett. 81, 3108 (1998).

[12] M.P.A. Fisher, P.B. Weichman, G. Grinstein, and D.S. Fisher, Phys. Rev. B, 40, 546 (1989).

[13] G.G. Batrouni, V. Rousseau, R.T. Scalettar, M. Rigol, A. Muramatsu, P.J.H. Denteneer, and M. Troyer, Phys. Rev. Lett. 89, 117203 (2002).

[14] F. Gerbier, A. Widera, S. Foelling, O. Mandel, T. Gericke and I. Bloch, Phys. Rev. A, 72, 053606 (2005)

[15] A. Isacsson, Min-Chul Cha, K. Sengupta, and S. M. Girvin, cond-mat/0508068 (2005).
[16] S. Fölling, A. Widera, T. Müller, F. Gerbier, and I. Bloch, cond-mat/0606592 (2006).

[17] F. Gerbier, S. Fölling, A. Widera, O. Mandel, and I. Bloch, Phys. Rev. Lett. 96, 090401 (2006).

[18] S.R. Clark and D. Jaksch, cond-mat/0604625 (2006).

[19] O. Penrose and L. Onsager, Phys. Rev. 104, 576 (1956).

[20] C. Orzel, A. K. Tuchman, M. L. Fenselau, M. Yasuda, and M. A. Kasevich, Science, 291, 2386 (2001).

[21] D. F. Walls and G. J. Milburn, Quantum Optics (Springer-Verlag, Berlin, 1994).

[22] C. M. Caves, Phys. Rev. D, 23, 1693 (1981).

[23] X. Lu and Y. Yu, cond-mat/0609230 (2006).

[24] N. V. Prokof'ev, B. V. Svistunov, and I. S. Tupitsyn, Phys. Lett. A, 238, 253 (1998); JETP 87, 310 (1998).

[25] G. Pupillo, C. J. Williams, and N. V. Prokof'ev, Phys. Rev. A, 73, 013408 (2006).

[26] T. Gericke, C. Utfeld, N. Hommerstad, and H. Ott, Laser Phys. Lett. 3, 415 (2006).

[27] see, e.g., E.M. Lifshitz and L.P. Pitaevskii, Statistical Mechanics, Part 2, Pergamon Press, New York, 1980.

[28] Yu. Kagan, B.V. Svistunov, and G.V. Shlyapnikov, Sov. Phys. - JETP 66, 314 (1987).

[29] B. Capagrosso-Sansone, N. V. Prokof'ev, and B. V. Svistunov, in preparation.

[30] W. Krauth and N. Triverdi, Europhys. Lett. 14, 627 (1991).

[31] V. A. Kashurnikov and B. V. Svistunov, Phys. Rev. B, 53, 11776 (1996).

[32] B. Paredes, A. Widera, V. Murg, O. Mandel, S. Fölling, I. Cirac, G. V. Shlyapnikov, T. W. Hänsch, and I. Bloch, Nature 429, 277 (2004).

[33] Fabrice Gerbier, private communication. 